\title{
Article
}

\section{The Incidence of Dichorionic Diamniotic Twin Pregnancy After Single Blastocyst Embryo Transfer and Zygosity: 8 Years of Single-Center Experience}

\author{
Hiroko Konno ${ }^{1}$, Takeshi Murakoshi ${ }^{1}$, Kiyonori Miura ${ }^{2}$ and Hideaki Masuzaki ${ }^{2}$ \\ ${ }^{1}$ Division of Obstetrics and Perinatology, Maternal and Perinatal Care Center, Seirei Hamamatsu General Hospital, Shizuoka, Japan and ${ }^{2}$ Obstetrics and \\ Gynecology, Nagasaki University School of Medicine, Nagasaki, Japan
}

\begin{abstract}
Dichorionic diamniotic (DCDA) twin pregnancies after single blastocyst embryo transfer have been reported recently, although a blastocyst ovum is generally believed to divide into monochorionic twin pregnancy. We investigated the incidence of DCDA twin pregnancy after single blastocyst embryo transfer and their zygosity. This prospective cohort study included 655 consecutive twin pregnancies that were managed from 2006 to 2014 at our institution. Chorionicity and amnionicity were determined using first-trimester ultrasonography and/or placental pathology. Zygosity was analyzed if the cases were DCDA twins after single blastocyst embryo transfer. Among 655 twin pregnancies, there were 348 DCDA cases, 295 monochorionic diamniotic (MCDA) cases and 12 monochorionic monoamniotic cases. Single blastocyst embryo transfer was performed in 43 cases. Six out of the 43 (14\%) cases involved DCDA twin pregnancies and the other 37 cases involved MCDA twin pregnancies. Three DCDA twins born after single blastocyst embryo transfer, wherein frozen embryo transfer (FET) was performed in the natural cycle, were dizygotic, and the other three cases, wherein FET with hormone replacement therapy was performed, were monozygotic. DCDA twin pregnancy occurred in 14\% (7\% for monozygotic and 7\% for dizygotic) of twin pregnancies after single blastocyst embryo transfer cases.
\end{abstract}

Keywords: Monozygotic dichorionic diamniotic twin; monochorionic diamniotic twin; single blastocyst embryo transfer; dizygotic; monozygotic

(Received 10 December 2019; accepted 18 December 2019; First Published online 25 March 2020)

Monozygotic dichorionic diamniotic (DCDA) twin pregnancies are believed to occur if the cleavage-stage embryo divides within 3 days of fertilization, before differentiation of the inner cell mass. Monochorionic diamniotic (MCDA) twin pregnancies occur if the blastocyst-stage embryo divides between days 4 and 8 postfertilization, at the time of differentiation of the inner cell mass. Monochorionic monoamniotic (MCMA) twin pregnancies occur if the embryo divides between days 9 and 12, when the amnion has become established (Knopman et al., 2010). A blastocyst ovum is generally believed to divide into monochorionic twin pregnancy.

However, recently, DCDA twin pregnancies after single blastocyst embryo transfer have been reported (Kyono, 2013; Shibuya \& Kyono, 2012; Sundaram et al., 2018; Van Langendonckt et al., 2000). Single blastocyst transfer is usually believed to result in MCDA or MCMA twin pregnancies, although it could result in DCDA twin pregnancies.

Among previous studies, there is only one known case wherein the zygosity in DCDA twin pregnancies after single blastocyst embryo transfer was analyzed accurately using genetic testing; the case was of a monozygotic DCDA twin pregnancy (Kyono, 2013).

Author for correspondence: Hiroko Konno, Email: hkonno@sis.seirei.or.jp Cite this article: Konno H, Murakoshi T, Miura K, and Masuzaki H. (2020) The Incidence of Dichorionic Diamniotic Twin Pregnancy After Single Blastocyst Embryo Transfer and Zygosity: 8 Years of Single-Center Experience. Twin Research and Human Genetics 23: 51-54, https://doi.org/10.1017/thg.2020.5
The mechanism of how a monozygotic DCDA twin pregnancy occurs by splitting a single blastocyst embryo is yet unknown. Processes such as micromanipulation of the zona pellucida during intracytoplasmic sperm injection (ICSI), preimplantation genetic screening (PGS) or preimplantation genetic diagnosis (PGD), or assisted hatching $(\mathrm{AH})$ might cause herniation and subsequent splitting of the inner cell mass. Furthermore, DCDA twin pregnancies after single blastocyst embryo transfer could occur through a combination of natural conception and embryo transfer, which is dizygotic.

The aim of this study was to estimate the incidence of DCDA twin pregnancies after single blastocyst embryo transfer and to analyze their zygosity of using genetic testing.

\section{Methods}

All consecutive twin pregnancies managed in our institution from 2006 to 2014 were included in this study. We studied how the patients became pregnant, and the chorionicity and amnionicity. Chorionicity and amnionicity were determined using first-trimester ultrasonography and/or postnatal placental pathology. A pregnancy was diagnosed as a DCDA twin pregnancy if a thick septum membrane between the two fetuses and the $\lambda$ sign was observed in ultrasonography, or two chorionic membranes and two amniotic membranes were observed in postnatal placental pathology. MCDA 
twin pregnancy was diagnosed if the following were observed: a thin septum membrane between two fetuses, consisting of two amniotic cavities, and the $\mathrm{T}$ sign on ultrasonography, or one chorionic membrane and two amniotic membranes were detected in postnatal placental pathology. MCMA twin pregnancy was diagnosed if the following were detected: no septum membrane between two fetuses on ultrasonography, or one chorionic membrane and one amniotic membrane on postnatal placental pathology.

Zygosity was analyzed if the cases were DCDA twins after a single blastocyst embryo transfer. Umbilical cord blood samples were obtained from the twin babies (baby 1 and baby 2) and whole blood samples were from their respective parents. Genomic DNA was extracted from the blood cells. Each sample was genotyped at 16 microsatellite markers using the AmpFISTR ${ }^{\circledR}$ Identifiler $^{\circledR}$ PCR Amplification Kit (Applied Biosystems, Foster City, CA, USA). DNA was amplified by PCR, and PCR products were analyzed using a 3100 Genetic Analyzer $^{\text {Tw }}$ (Applied Biosystems, Foster City, CA, USA). At informative loci showing a discordant genotype between parents, all twin babies inherited one copy each of alleles from their respective parents. When there were discordant genotypes at one or more informative loci, twins were diagnosed as dizygotic (Case 2, Figure 1). When genotypes of baby 1 and baby 2 were identical at all informative loci, twins were diagnosed as a monozygotic (Case 4, Figure 1).

This study was approved by the ethics committee and the institutional research review board. Informed consent was sought and received from participating mothers. The data analysis was carried out according to the principles of the Declaration of Helsinki.

\section{Results}

Among the 655 twin pregnancies, there were 348 DCDA cases, 295 MCDA cases and 12 MCMA cases. The characteristics of all cases are shown in Table 1. Assisted reproduction was performed in 130 cases. Blastocyst embryo transfer was performed in 108 cases, among which 43 were single blastocyst embryo transfer. The characteristics of single blastocyst transfer are shown in Table 2. Six out of these 43 (14\%) were DCDA twin pregnancies and the other 37 cases were MCDA twin pregnancies (Figure 2).

The zygosities of all six DCDA twins after single blastocyst embryo transfer were analyzed by means of the combined DNA index system. The characteristics of these six cases are shown in Table 3. Three cases were dizygotic and the other three cases were monozygotic DCDA twin pregnancies (Figure 1).

\section{Discussion}

DCDA twin pregnancies are either of monozygotic or dizygotic origin. DCDA twin pregnancies after single-embryo transfer are believed to result from separate cleavage-stage embryos until day 3 after fertilization (Knopman et al., 2010). However, DCDA twin pregnancies after single blastocyst embryo transfer have been reported (Kyono, 2013; Shibuya \& Kyono, 2012; Sundaram et al., 2018; Van Langendonckt et al., 2000). Single blastocyst transfer is believed to result in MCDA or MCMA twin pregnancies, although it could result in DCDA twin pregnancies as well.

In previous studies, 12 multichorionic cases occurring after single blastocyst transfer have been reported (Sundaram et al., 2018). There has been only one case where zygosity was confirmed using genetic testing in DCDA twin pregnancies after a single blastocyst embryo transfer, and this case was a monozygotic pregnancy (Kyono, 2013). To the best of our knowledge, there are no
Table 1. Maternal characteristics in all cases

\begin{tabular}{lccc} 
& $\begin{array}{c}\text { Natural } \\
n=407\end{array}$ & $\begin{array}{c}\text { COS } \\
n=118\end{array}$ & $\begin{array}{c}\text { ART } \\
n=130\end{array}$ \\
\hline Age (years) & $29.9 \pm 5.0$ & $31.6 \pm 4.0$ & $34.5 \pm 4.5$ \\
\hline Primipara & $200(49 \%)$ & $74(63 \%)$ & $106(82 \%)$ \\
\hline BMI & $20.5 \pm 2.9$ & $21.1 \pm 3.3$ & $20.8 \pm 3.2$ \\
\hline Body weight $(\mathrm{kg})$ & $51.4 \pm 7.7$ & $52.7 \pm 9.3$ & $51.9 \pm 7.5$ \\
\hline MCMA & $10(2 \%)$ & $2(2 \%)$ & $0(0 \%)$ \\
\hline MCDA & $237(58 \%)$ & $19(16 \%)$ & $39(30 \%)$ \\
\hline DCDA & $160(39 \%)$ & $97(82 \%)$ & $91(70 \%)$ \\
\hline
\end{tabular}

Note: Data are presented as mean \pm SD or number (\%).

$\mathrm{COS}$, controlled ovarian stimulation; ART, assisted reproductive technology; BMI, body mass index; MCMA, monochorionic monoamniotic twin; MCDA, monochorionic diamniotic twin; DCDA, dichorionic diamniotic twin.

Table 2. Maternal characteristics of single blastocyst transfer

\begin{tabular}{lcc}
\hline & $\begin{array}{c}\text { DCDA } \\
n=6\end{array}$ & $\begin{array}{l}\text { MCDA } \\
n=37\end{array}$ \\
\hline Age (years) & $33.2 \pm 3.1$ & $34.4 \pm 4.2$ \\
\hline Primipara & $6(100 \%)$ & $27(73 \%)$ \\
\hline BMI & $19.3 \pm 1.6$ & $20.8 \pm 2.5$ \\
\hline Body weight (kg) & $46.6 \pm 5.4$ & $51.7 \pm 6.5$ \\
\hline Gestational weeks at delivery & $37(37-38)$ & $37(17-39)$ \\
\hline
\end{tabular}

Note: Data are presented as mean $\pm \mathrm{SD}$, median (range) or number (\%).

DCDA, dichorionic diamniotic twin; MCDA, monochorionic diamniotic twin; BMI, body mass index.

studies that have accurately analyzed the incidence of zygosity of multichorionic cases after single blastocyst transfer using genetic testing.

In this study, 43 cases underwent single blastocyst embryo transfer. Furthermore, the zygosities of 6 DCDA twin pregnancies among these 43 twins were analyzed using the combined DNA index system. Three cases were confirmed to be dizygotic and the other three cases were confirmed to be monozygotic. This indicates that the occurrence of monozygotic DCDA twin pregnancies by single blastocyst transfer was the result of a separate blastocyst, and the occurrence of dizygotic DCDA twin pregnancies by single blastocyst transfer was pregnancies that occurred due to natural ovulation and concurrent blastocyst transfer. In three dizygotic cases, frozen embryo transfer (FET) was performed in the natural cycle without hormone supplements, which meant that spontaneous ovulation could have occurred. Dizygotic cases could be explained by a single embryo with a concurrent natural conception. On the other hand, in three monozygotic cases, FET was performed with hormone replacement therapy, which means that spontaneous ovulation could not have occurred. Monozygotic cases can be explained only by splitting of a single blastocyst embryo.

The mechanism of monozygotic DCDA twins by splitting a single blastocyst embryo has been inferred in several studies. A previous study reported visualization of an atypical hatching pattern of a vitrified or warmed blastocyst that resulted in two separate and complete blastocysts in vitro using time-lapse cinematography (Nishimura et al., 2014). A frozen-thawed blastocyst could split into two separate blastocysts and escape the zona pellucida. 
Table 3. Maternal characteristics of DCDA twins by single blastocyst transfer

\begin{tabular}{|c|c|c|c|c|c|c|}
\hline & Case 1 & Case 2 & Case 3 & Case 4 & Case 5 & Case 6 \\
\hline Age (years) & 29 & 30 & 36 & 35 & 33 & 36 \\
\hline Parity & 0 & 0 & 0 & 0 & 0 & 0 \\
\hline BMI & 19.4 & 21.2 & 21.1 & 17.3 & 17.7 & 19.0 \\
\hline ET & Natural & Natural & Natural & HRT & HRT & HRT \\
\hline Oocyte age (years) & 28 & 29 & 36 & 34 & 32 & 35 \\
\hline Micromanipulation & None & None & ICSI & ICSI & None & ICSI \\
\hline Frozen or fresh & Frozen & Frozen & Frozen & Frozen & Frozen & Frozen \\
\hline Gestational weeks at first visit & 12 & 10 & 9 & 12 & 10 & 10 \\
\hline Gestational weeks at delivery & 33 & 37 & 37 & 38 & 38 & 36 \\
\hline Fetal weights (g) & $1886 / 1882$ & $2558 / 2626$ & $2626 / 2914$ & $2548 / 2754$ & $2712 / 2602$ & $2502 / 2352$ \\
\hline Fetal sex & $M / M$ & $M / F$ & $M / M$ & $\mathrm{~F} / \mathrm{F}$ & $M / M$ & $M / M$ \\
\hline Zygocity & D & $\mathrm{D}$ & D & M & M & $M$ \\
\hline
\end{tabular}

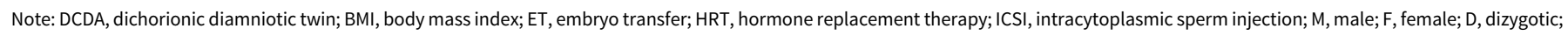
$\mathrm{M}$, monozygotic.

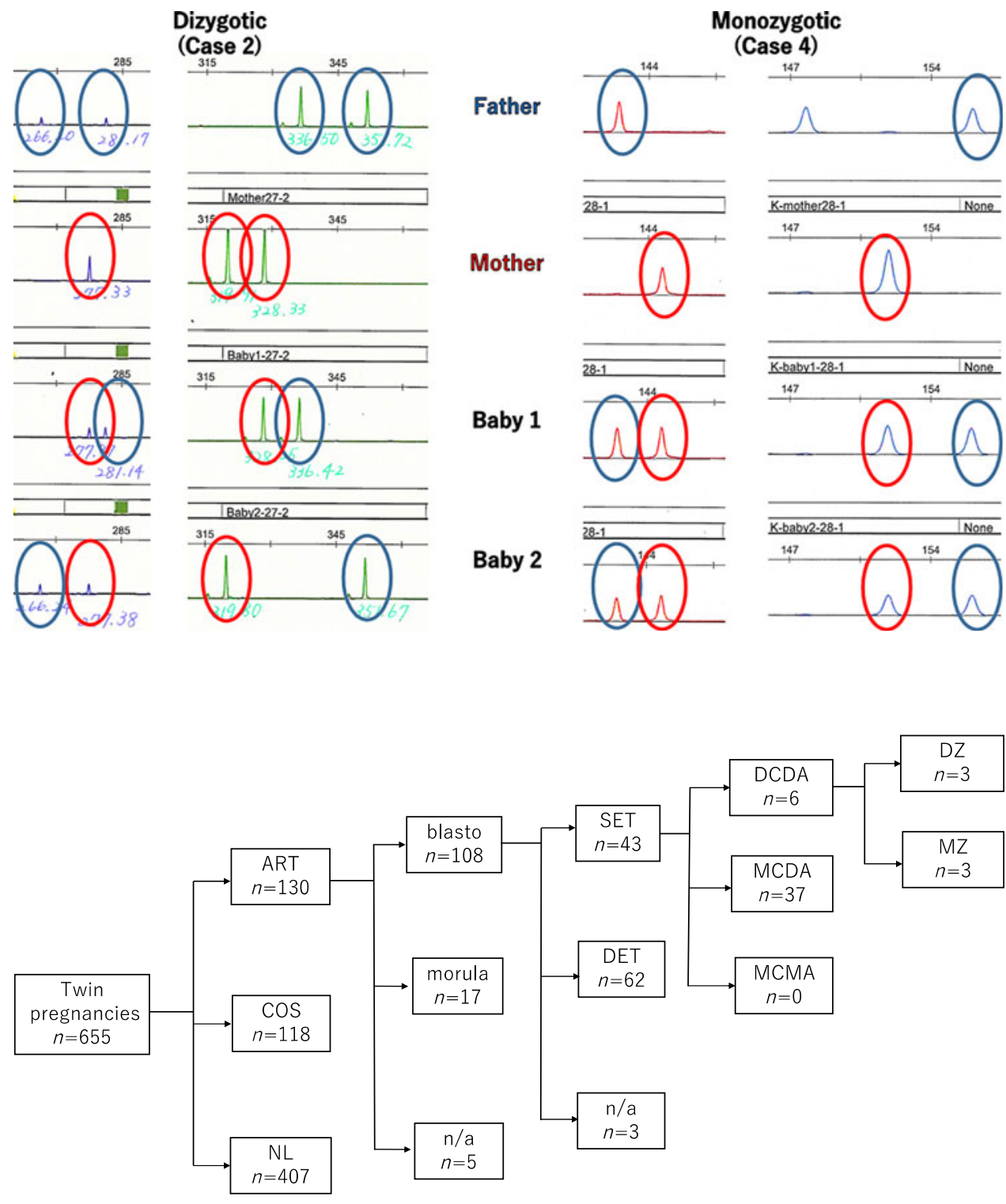

Fig. 1. Determination of twin zygosity by DNA genotyping at microsatellite marker loci. When there were discordant genotypes at one or more informative loci, twins were diagnosed as dizygotic (Case 2). When genotypes of baby 1 and baby 2 were identical at all informative loci showing a discordant genotype between parents, twins were diagnosed as a monozygotic (Case 4).
Fig. 2. Overview of all cases.

Note: ART, assisted reproductive technology; COS, controlled ovarian stimulation; NL, natural; $\mathrm{SET}$, single-embryo transfer; DET, doubleembryo transfer; DCDA, dichorionic diamniotic twin; MCDA, monochorionic diamniotic twin; MCMA, monochorionic monoamniotic twin; DZ, dizygotic; MZ, monozygotic. 
Some studies have reported that micromanipulation of the zona pellucida during ICSI, PGS/PGD or AH might cause herniation and subsequent splitting of the inner cell mass (Alikani et al., 1994, Elizur et al., 2004; Hershlag et al., 1999; Nakasuji et al., 2014). In this study, micromanipulation was done in two cases of three monozygotic DCDA twin pregnancies. Although not all cases can be explained by micromanipulation, the relation cannot be denied.

Three cases (7\%) of 43 single blastocyst transfer were monozygotic DCDA cases. In our study, the incidence of monozygotic DCDA twin pregnancies after single blastocyst transfer was not rarer than expected. Some studies have reported that the risk factors of monozygotic twin pregnancy after single-embryo transfer are young oocyte age, extended embryo culture and micromanipulation, such as ICSI or AH (Busnelli et al., 2019). In this study, blastocyst transfer was found to likely increase the incidence of monozygotic twin pregnancy after in vitro fertilization, which is comparable to the results of prior studies.

This study had several limitations, namely that we reported a small number of DCDA twin pregnancies by single blastocyst transfer, and not all the cases we studied underwent assisted reproductive technology in our institution. We need to expand the study to multiple centers in Japan and possibly in different countries. However, the primary strength of this study was that all DCDA twin pregnancies by single blastocyst transfer underwent genetical analysis of their zygosity using the combined DNA index system.

In conclusion, DCDA twin pregnancies were found to occur in six (14\%) cases of twin pregnancies after single blastocyst embryo transfer, three (7\%) cases of dizygotic pregnancies and three (7\%) cases of monozygotic pregnancies. Dizygotic DCDA twin pregnancies after single blastocyst embryo transfer can result from a combination of natural conception and embryo transfer. A monozygotic DCDA twin pregnancy can also exist commonly after a single blastocyst embryo transfer, although the splitting mechanism is unknown.

Financial support. This research received no specific grant from any funding agency, commercial or not-for-profit sectors.

Conflict of interest. None.

Ethical standards. The authors assert that all procedures contributing to this work comply with the ethical standards of the relevant national and institutional committees on human experimentation and with the Helsinki Declaration of 1975, as revised in 2008. The authors assert that all procedures contributing to this work comply with the ethical standards of the relevant national and institutional guides on the care and use of laboratory animals.

\section{References}

Alikani, M., Noyes, N., Cohen, J., \& Rosenwaks, Z. (1994). Monozygotic twinning in the human is associated with the zona pellucida architecture. Human Reproduction, 9, 1318-1321.

Busnelli, A., Dallagiovanna, C., Reschini, M., Paffoni, A., Fedele, L., \& Somigliana, E. (2019). Risk factors for monozygotic twinning after in vitro fertilization: A systematic review and meta-analysis. Fertility Sterility, 111, 302-317.

Elizur, S. E., Levron, J., Shrim, A., Sivan, E., Dor, J., \& Shulman, A. (2004). Monozygotic twinning is not associated with zona pellucida micromanipulation procedures but increases with high-order multiple pregnancies. Fertility Sterility, 82, 500-501.

Hershlag, A., Paine, T., Cooper, G. W., Scholl, G. M., Rawlinson, K., \& Kvapil, G. (1999). Monozygotic twinning associated with mechanical assisted hatching. Fertility Sterility, 71, 144-146.

Knopman, J., Krey, L. C., Lee, J., Fino, M. E., Novetsky, A. P., \& Noyes, N. (2010). Monozygotic twinning: An eight-year experience at a large IVF center. Fertility Sterility, 94, 502-510.

Kyono, K. (2013). The precise timing of embryo splitting for monozygotic dichorionic diamniotic twins: When does embryo splitting for monozygotic dichorionic diamniotic twins occur? Evidence for splitting at the morula/ blastocyst stage from studies of in vitro fertilization. Twin Research and Human Genetics, 16, 827-832.

Nakasuji, T., Saito, H., Araki, R., Nakaza, A., Nakashima, A., Kuwahara, A., ..., Sakumoto, T. (2014). The incidence of monozygotic twinning in assisted reproductive technology: Analysis based on results from the 2010 Japanese ART national registry. Journal of Assisted Reproduction and Genetics, 31, 803-807.

Nishimura, M., Tsuchiya, S., Kaneko, Y., Matsui, Y., Iizumi, A., Sato, K., \& Araki, Y. (2014). Observation of a frozen-thawed blastocyst dividing via time-lapse cinematography and a possible mechanism for a dichorionicdiamniotic pregnancy. The Journal of Clinical Embryology, 17, 191-195.

Shibuya, Y., \& Kyono, K. (2012). A successful birth of healthy monozygotic dichorionic diamniotic (DD) twins of the same gender following a single vitrified-warmed blastocyst transfer. Journal of Assisted Reproduction and Genetics, 29, 255-257.

Sundaram, V., Ribeiro, S., \& Noel, M. (2018). Multi-chorionic pregnancies following single embryo transfer at the blastocyst stage: A case series and review of the literature. Journal of Assisted Reproduction and Genetics, 35, 2109-2117.

Van Langendonckt, A., Wyns, C., Godin, P. A., Toussaint-Demylle, D., \& Donnez, J. (2000). Atypical hatching of a human blastocyst leading to monozygotic twinning: A case report. Fertility Sterility, 74, 1047-1050. 